\title{
Can Space Syntax Help Us in Understanding the Intraurban Office Rent Pattern? Accessibility and Rents in Downtown Stockholm
}

\section{Rickard Enström • Olof Netzell}

Published online: 25 July 2008

(C) Springer Science + Business Media, LLC 2008

\section{Erratum to: J Real Estate Finance Econ (2008) 36:289-305 DOI 10.1007/s11146-007-9054-6}

The original version of this article unfortunately contained a mistake. The presentation of Table 3 was incorrect due to missing information. The corrected table is given below.

Table 3 Regression on $\log$ of the rent, 1999-2002; translog specification

All explanatory variables are in $\log$ form

$\alpha_{i j}$ and $\alpha_{j i}$ in (23) are assumed equal for $i \neq j$

Interaction variables have been halved

\begin{tabular}{|c|c|c|c|c|}
\hline Variable & Coeff. & Std. error & $t$ value & $\begin{array}{l}\text { Level of } \\
\text { significance }\end{array}$ \\
\hline Intercept & 5.994 & 1.246 & 4.810 & 0.000 \\
\hline Size & 0.882 & 0.180 & 4.889 & 0.000 \\
\hline Dist & 0.645 & 0.236 & 2.733 & 0.007 \\
\hline Integration & 6.426 & 2.224 & 2.890 & 0.004 \\
\hline $\operatorname{Size}^{2}$ & -0.005 & 0.020 & -0.273 & 0.785 \\
\hline Dist $^{2}$ & -0.142 & 0.028 & -4.992 & 0.000 \\
\hline Integration $^{2}$ & -2.936 & 3.686 & -0.796 & 0.427 \\
\hline Size_Dist & 0.061 & 0.037 & 1.634 & 0.104 \\
\hline Size_Integration & -0.287 & 0.387 & -0.741 & 0.460 \\
\hline Dist_Integration & -1.289 & 0.556 & -2.317 & 0.022 \\
\hline Contract_long & 0.082 & 0.055 & 1.482 & 0.140 \\
\hline Contract_three & 0.077 & 0.050 & 1.527 & 0.129 \\
\hline$H Y_{99: 2}$ & -0.021 & 0.073 & -0.285 & 0.776 \\
\hline$H Y_{00: 1}$ & 0.285 & 0.060 & 4.781 & 0.000 \\
\hline$H Y_{00: 2}$ & 0.385 & 0.064 & 5.975 & 0.000 \\
\hline$H Y_{01: 1}$ & 0.392 & 0.061 & 6.466 & 0.000 \\
\hline$H Y_{01: 2}$ & 0.423 & 0.069 & 6.118 & 0.000 \\
\hline$H Y_{02: 1}$ & 0.440 & 0.067 & 6.533 & 0.000 \\
\hline$H Y_{02: 2}$ & 0.420 & 0.093 & 4.506 & 0.000 \\
\hline \multicolumn{5}{|l|}{$n=184$} \\
\hline$R^{2}$ & 0.971 & & & \\
\hline Adj. $R^{2}$ & 0.968 & & & \\
\hline$F$ & 308.255 & & & 0.000 \\
\hline Variance of the estimate & 0.0417 & & & \\
\hline Sum of squared errors & 6.8773 & & & \\
\hline
\end{tabular}

The online version of the original article can be found at http://dx.doi.org/10.1007/s11146-007-9054-6.

R. Enström $(\bowtie)$

School of Business, University of Alberta, Edmonton, AB, Canada T6G 2R6

e-mail: rickard.enstrom@ualberta.ca

O. Netzell

Building and Real Estate Economics, KTH, 10044 Stockholm, Sweden e-mail: netzell@kth.se

를 Springer 